${ }^{3}$ Melbourne Medical School, Faculty of Medicine, Dentistry and Health Sciences, Melbourne, Australia

${ }^{4}$ Department of Endocrinology, Osijek University Hospital, Osijek, Croatia

${ }^{5}$ Department of Internal Medicine, Faculty of Medicine, J. J. Strossmayer University of Osijek, Croatia

${ }^{6}$ Institute of Clinical Laboratory Diagnostics, Osijek University Hospital, Osijek, Croatia

${ }^{7}$ Institute "Prof. Radivoje Radić", Faculty of Dental Medicine and Health Osijek, J. J. Strossmayer University of Osijek, Croatia

\title{
Magnesium, zinc and iron serum levels as potential parameters significant for effective glycemic control in children with type 1 diabetes
}

\section{ABSTRACT}

Background. Various trace elements contribute to the development of diabetes and its complications through their roles in glucose metabolism and the oxidative stress response. The aim of this study was to ascertain the difference in serum magnesium, zinc and iron concentrations between healthy children and children with type 1 diabetes mellitus (T1DM). This study also aimed to determine whether serum concentrations of magnesium, zinc, and iron in children with T1DM correlated with the duration of the disease and the quality of glycemic control in this group.

Material and methods. A total of 99 children with T1DM and $\mathbf{4 0}$ healthy children were included in this study. Magnesium, zinc and iron serum levels were assessed using the photometric method.

Address for correspondence:

Robert Lovrić, PhD, MSN, Assistant professor

Institute "Prof. Radivoje Radić"

Faculty of Dental Medicine and Health

J. J. Strossmayer University of Osijek

Crkvena 21a, Osijek, 31000, Croatia

Phone: +385-91-2000-973

Fax: +385-31-399-601

e-mail: rlovric@mefos.hr

Clinical Diabetology 2020, 9, 3, 161-166

DOI: $10.5603 /$ DK.2020.0014

Received: 31.01 .2020
Results. Significantly lower levels of magnesium and zinc ( $P<0.001$ ) were observed, between the T1DM group and the healthy control group but no statistically significant differences were found in iron levels $(P=0.13)$ between the two groups. While there were no statistically significant differences in serum concentrations with respect to the duration of disease, it was, however, discovered that children with poorer glycemic control had significantly lower serum zinc concentrations ( $P<0.001)$ while magnesium and iron levels remained similar ( $P=0.07$ and 0.21 respectively). Conclusion. This study found that while there was no significant difference in iron serum levels in children with T1DM compared to healthy controls, children with T1DM did have more significantly decreased magnesium and zinc serum levels than the control group. Serum zinc levels in this study also directly correlated to poorer glycemic control. Further studies are required to explore whether magnesium and zinc supplementation, or nutritional intake, could potentially be used to achieve better glycemic control in children with T1DM. (Clin Diabetol 2020; 9; 3: 161-166)

Key words: iron, magnesium, zinc, diabetes mellitus, type 1 , child, supplements

\section{Introduction}

Despite continuous efforts and rapid technological as well as pharmaceutical advances, diabetes mel- 
litus continues to be one of the most common health problems in the general population. Children with type 1 diabetes mellitus (T1DM) are especially interesting patient group, because any findings concerning the mechanisms of diabetes and the development of diabetic complications in these children can be used to improve their future quality of life. In view of that, recent studies have explored the effect of various elements in the formation of oxygen-free radicals as well as the effect of the ensuing oxidative stress on the development of diabetic complications [1, 2].

Magnesium is involved in the synthesis of glutathione, a major antioxidant, through ensuring the proper functioning of the enzyme gamma glutamyl transpeptidase (GGT) [3]. It also plays a vital role in glucose metabolism, and its deficiency has been most commonly associated with diabetes mellitus [4] through its effect on the development of dyslipidaemia, increased insulin resistance and carbohydrate intolerance [5]. Hypomagnesemia also increases the possibility of developing diabetic complications, particularly atherosclerosis [6], diabetic retinopathy [7] and end-stage renal disease (ESRD) [8]. Another important element in glucose metabolism is zinc ( $\mathrm{Zn})$, which plays a role in secretion of insulin from pancreatic cells [9] where they form a Zn-insulin complex [2]. It further increases hepatic binding of insulin, as well as having a role in the modulation of insulin actions. As an antioxidant, zinc stabilizes membranes and reduces hydroxyl radicals $(\mathrm{OH})$ through the induction of metallothionein synthesis [10]. Both of these functions are diminished in cases of zinc deficiency, which has impacts on glycaemic control. In children with T1DM, it has been observed that Zn deficiency directly correlates with poorer glycemic control and increased $\mathrm{HbA}_{1 \mathrm{c}}$ [11].

In regards to serum iron levels, iron deficiency has been correlated with an increased $\mathrm{HbA}_{1 \mathrm{c}}$ [12] in older patients with T1DM, although the same correlation was not noted in most children with diabetes [13]. Children with newly discovered T1DM, however, were found to have lower serum levels of iron than children who have had T1DM for a number of years [14]. Therefore, both increased and decreased serum levels of iron may have possible negative outcomes in patients with T1DM. On one hand, iron deficiency causes microcytic anemia, which is common in patients with T1DM and is associated with the progression of diabetes, as well as the development of its co-morbidities such as micro- and macroangiopathies [15]. On the other hand, through the Fenton reaction, increased serum levels of iron can generate reactive oxygen species, damaging tissues or cells [16].
Since various elements are closely linked to the progression of diabetes and its co-morbidities, the aim of this study was to ascertain the difference in serum magnesium, zinc and iron concentrations between healthy children and children with T1DM. This study also aimed to determine whether serum concentrations of magnesium, zinc, and iron in children with T1DM correlated with the duration of disease and the quality of glycemic control in this group.

By fulfilling these aims, this study could help increase understanding about the mechanisms of magnesium, zinc and iron involvement in T1DM, as well the possibilities of supplementing those elements to ensure better glycemic control in children with T1DM.

\section{Material and methods Subjects}

In this case-control study, a total of 139 children under 18 years of age were included, among which 99 children had previously diagnosed T1DM, while 40 represented a healthy control group. The control group was comprised of children who had undergone a standard systematic examination in a paediatric practice, and were not suffering from diabetes mellitus, acute infections, malignant, autoimmune or chronic diseases. For the purpose of this study the following tests were performed to rule out underlying diseases in the control group: DM was excluded in the control group based on blood glucose measurements (fasting plasma glucose $<5.6 \mathrm{mmol} / \mathrm{l}$ ) and the absence of DM symptoms [17]. Acute infections were excluded by complete physical examination and normal laboratory values of white blood cells (WBC) and C-reactive protein (CRP). The T1DM group consisted of children that satisfied the criteria defined by the International Society for Paediatric and Adolescent Diabetes (ISPAD) Clinical Practice Consensus Guidelines 2018 Compendium [18]. Blood glucose levels, levels of islet cell autoantibodies (ICA), glutamic acid decarboxylase 65 (GAD-65) autoantibodies, and insulinoma-associated protein-2 (IA-2), as well as the presence of usual symptoms accompanying T1DM were used to determine the diagnosis of T1DM. Children with T1DM who were suffering from associated autoimmune diseases (such as celiac disease or Hashimoto thyroiditis) were not included in this study. In children with T1DM, serum levels of magnesium, zinc and iron were compared and correlated with the duration of disease and the quality of disease control. Regarding T1DM duration, children were grouped as either duration of T1DM under or over 1 year [17]. Furthermore, in accordance with the criteria defined by ISPAD [18], in children with long lasting disease 
( $>1$ year), glycemic control was defined as either good $\left(\mathrm{HbA}_{1 \mathrm{c}} \leq 7 \%\right)$ or poor glycemic control $\left(\mathrm{HbA}_{1 \mathrm{c}}>7 \%\right)$.

\section{Methods}

Blood samples were collected in two tubes. Clot activator tubes (Becton, Dickinson, and Company, Franklin Lakes, NJ, USA) were used for glucose, as well as for serum zinc, iron and magnesium levels. The coefficients of variation (CV \%) for these elements were as follows: for $\mathrm{Zn}$ within-run (1.7), between-run (2.1) and reference values (10.7-18.4 $\mu \mathrm{mol} / \mathrm{l})$; for $\mathrm{Mg}$ within-run (1.02), between-run (1.15) and reference values $(0.74-0.97 \mathrm{mmol} / \mathrm{l})$; for Fe within-run (1.02), between-run (2.09) and reference values (6-31 $\mu \mathrm{mol} / \mathrm{l})$. Samples were centrifuged for 10 minutes at $1500 \mathrm{~g}$. K2EDTA tubes (Becton, Dickinson, and Company, Franklin Lakes, NJ, USA) were used for WBC count and haemoglobin $A_{1 c}\left(\mathrm{HbA}_{1 \mathrm{c}}\right)$ measurement. Blood samples were collected in the fasting state according to the recommendations of the Clinical Laboratory Standard Institute (CLSI) between 7:00 and 9:00 a.m. [19]. All tests were performed immediately. CRP was measured by immunoturbidimetric assay (Roche Diagnostics $\mathrm{GmbH}$, Mannheim, Germany), glucose was measured by the enzymatic method (Beckman Coulter Inc., Brea, USA), and zinc, magnesium as well as iron serum levels were measured with the photometric method using Beckman Coulter AU680 analyser (Beckman Coulter Inc., Brea, USA). WBC count was determined using a Sys-mex SF-3000 automated haematology analyser (Sysmex Corporation, Kobe, Japan) while $\mathrm{HbA}_{1 \mathrm{c}}$ was determined by the immunoturbidimetric method on a Dimension EXL with an LM analyser (Siemens Healthcare Diagnostics Inc., USA).

\section{Statistical analysis}

Data were reported using descriptive statistical methods. The Mann-Whitney $U$ test was used to compare the medians between the two groups. All $P$ values are two-sided. The level of significance is set at alpha $=$ 0.05 . Statistical analysis was performed by the statistical program MedCalc Statistical Software version 19.0.5 (MedCalc Software bvba, Ostend, Belgium; https:// www.medcalc.org; 2019).

\section{Ethical statement}

Informed consent was obtained from parents or legal guardians of all children enrolled in the study. The study was performed in accordance with the Declaration of Helsinki and was approved by the Ethics Committee of Osijek University Hospital and the Faculty of Medicine, University of Osijek (12 March, 2018, IRB number: 2158-61-07-18-14).
Table 1. Distribution of respondents by gender in studied groups $(\mathrm{N}=139)$

\begin{tabular}{lcccc}
\hline Gender & \multicolumn{2}{c}{ N (\%) } & Total & P-value $^{\dagger}$ \\
\cline { 2 - 3 } & $\begin{array}{c}\text { Control } \\
\text { group }\end{array}$ & $\begin{array}{c}\text { T1DM* } \\
\text { group }\end{array}$ & & \\
\hline Boys & $18(45.0)$ & $47(47.5)$ & $65(46.8)$ & 0.85 \\
Girls & $22(55.0)$ & $52(52.5)$ & $74(53.2)$ & \\
Total & $40(100)$ & $99(100)$ & $139(100)$ & \\
\hline
\end{tabular}

*T1DM group - children with type 1 diabetes mellitus; ${ }^{\dagger}$ Fisher exact test

Table 2. Demographic and clinical characteristics of study groups $(\mathrm{N}=136)$

\begin{tabular}{|c|c|c|c|}
\hline \multirow[t]{2}{*}{ Factor } & \multicolumn{2}{|c|}{ Median (interquartile range) } & \multirow[t]{2}{*}{ P-value ${ }^{\dagger}$} \\
\hline & $\begin{array}{l}\text { Control } \\
\text { group }\end{array}$ & $\begin{array}{l}\text { T1DM* } \\
\text { group }\end{array}$ & \\
\hline Age (years) & $13(13.0-17.0)$ & $12(8.0-14.0)$ & 0.09 \\
\hline BMI $\left[\mathrm{kg} / \mathrm{m}^{2}\right]$ & $16.9(15.1-18.1)$ & $17.1(14.9-21.3)$ & 0.11 \\
\hline $\mathrm{HbA}_{1 \mathrm{c}}(\%)$ & $5.1(4.7-5.5)$ & $7.9(7.2-8.6)$ & 0.03 \\
\hline
\end{tabular}

$\mathrm{BMI}$ - body mass index; $\mathrm{HbA}_{1 \mathrm{c}}$ - haemoglobin $\mathrm{A}_{1 \mathrm{c}} ;{ }^{*} \mathrm{~T} 1 \mathrm{DM}$ group children with type 1 diabetes mellitus; ${ }^{\dagger}$ Mann-Whitney $U$ test

\section{Results}

This case control study included 139 children, of which 99 (71.2\%) were diagnosed with T1DM and 40 (29.5\%) who were used as a healthy control group after ruling out T1DM, acute infections or other underlying diseases. There were 74 girls (53.2\%) and 65 boys (46.8\%) with no significant difference between the groups (Table 1).

The mean age of children in the two groups was 14 years (Table 2). Table 2 further describes the characteristics of the study groups (age, $\mathrm{BMI}$ and $\mathrm{HbA}_{1 \mathrm{c}}$ ).

In children with T1DM, the median (interquartile range) of disease duration was 4.1 (3.9-4.3) years. Out of the total number $(\mathrm{N}=99)$ of children with T1DM, 10 (9.9\%) children were treated with an insulin pump, while the other $89(90.1 \%)$ children used intensive insulin therapy. In comparing the two groups, it was shown that the T1DM group had significantly lower levels of both magnesium ( $P<0.001)$, and zinc $(P<0.001)$ than the control group. However, there was no significant difference in measured serum iron levels between the two groups (Table 3).

With respect to the duration of disease within the T1DM group, there were no significant differences in serum magnesium, zinc and iron levels (Table 4).

Significantly lower zinc serum levels were measured in children with poorer glycemic control $(P<0.001)$. However, no statistically significant difference was observed in serum iron and magnesium levels (Table 5). 
Table 3. Differences in serum magnesium, zinc and iron levels in studied groups $(N=139)$

\begin{tabular}{|c|c|c|c|c|c|}
\hline \multirow[t]{2}{*}{ Elements } & \multicolumn{2}{|c|}{ Median (interquartile range) } & \multirow[t]{2}{*}{ HL diff. } & \multirow[t]{2}{*}{$95 \% \mathrm{Cl}$} & \multirow[t]{2}{*}{ P-value } \\
\hline & Control & T1DM & & & \\
\hline Magnesium [mg] & $0.87(0.83-0.90)$ & $0.80(0.75-0,84)$ & -0.08 & -0.1 to -0.05 & $<0.001 *$ \\
\hline Zinc $[\mathrm{mmol} / \mathrm{L}]$ & $12.9(11.8-13.8)$ & $10.7(9.3-12.1)$ & -2.1 & -2.7 to -1.4 & $<0.001 *$ \\
\hline Iron $[\mu \mathrm{mol} / \mathrm{L}]$ & $15.9(10.6-20.5)$ & $14.4(9.7-18.03)$ & -1.7 & -4.1 to 0.6 & 0.13 \\
\hline
\end{tabular}

HL diff. — Hodges-Lehmann median difference; *significant at $\mathrm{P} \leq 0.05$

Table 4. Comparison of serum magnesium, zinc and iron levels in children with T1DM $(N=99)$ in regard to the duration of T1DM

\begin{tabular}{|c|c|c|c|c|c|}
\hline \multirow[t]{2}{*}{ Elements } & \multicolumn{2}{|c|}{ Median (interquartile range) } & \multirow[t]{2}{*}{ HL diff. } & \multirow[t]{2}{*}{$95 \% \mathrm{Cl}$} & \multirow[t]{2}{*}{ P-value } \\
\hline & Under 1 year $(n=7)$ & Over 1 year $(n=92)$ & & & \\
\hline Magnesium [mg] & $0.75(0.72-0.79)$ & $0.70(0.75-0.84)$ & 0.04 & -0.02 to 0.09 & 0.12 \\
\hline Zinc $[\mathrm{mmol} / \mathrm{L}]$ & $10.7(10.2-11.1)$ & $10.7(9.2-12.1)$ & -0.3 & -1.6 to 1.2 & 0.73 \\
\hline Iron $[\mu \mathrm{mol} \mathrm{L}]$ & $15.4(12.4-17.8)$ & $13.9(9.7-18.1)$ & -1.5 & -5.5 to 2.6 & 0.45 \\
\hline
\end{tabular}

HL diff. - Hodges-Lehmann median difference

Table 5. Comparison of serum magnesium, zinc and iron levels in children with T1DM ( $>1$ year) according to the quality of glycemic control in ( $\mathrm{N}=92)$

\begin{tabular}{lcccccc}
\hline \multirow{2}{*}{ Elements } & \multicolumn{2}{c}{ Median (interquartile range) } & HL diff. & 95\% Cl & P-value \\
\cline { 2 - 3 } & $\mathbf{H b A}_{1 \mathrm{c}} \leq \mathbf{7 \%}(\mathbf{n}=\mathbf{4 8})$ & $\mathbf{H b A}_{1 \mathrm{c}}>\mathbf{7 \%}(\mathbf{n}=\mathbf{4 4 )}$ & & & & \\
\hline Magnesium $[\mathrm{mg}]$ & $0.82(0.76-0.85)$ & $0.79(0.75-0.82)$ & -0.02 & -0.04 to 0.01 & 0.21 \\
Zinc $[\mathrm{mmol} / \mathrm{L}]$ & $11.2(10.2-13.1)$ & $9.9(8.8-11.2)$ & -1.4 & -2.2 to -0.6 & $<0.001^{*}$ \\
Iron $[\mu \mathrm{mol} / \mathrm{L}]$ & $15.2(10.9-18.8)$ & $11.5(9.6-16.5)$ & -2.3 & -4.7 to 0.10 & 0.07 \\
\hline
\end{tabular}

HL diff. - Hodges-Lehmann median difference; ${ }^{*}$ significant at $\mathrm{P} \leq 0.05$

\section{Discussion}

In recent times, T1DM in paediatric patients has become one of the most researched endocrinological disorders in the world. Among the many things studied, the correlation between the imbalance of trace elements in the human body and their effects on the severity of T1DM through the effects of oxidative stress is particularly interesting [2]. It has been suggested that achieving the balance of various trace elements in the body could improve glycemic control in T1DM patients by using relatively simple means of pharmaceutical and nutritional correction. Magnesium and zinc are the obvious potential candidates due to their important roles in glucose metabolism and antioxidant response.

The results of this study demonstrate significantly lower serum levels of magnesium and zinc in children with T1DM when compared to healthy controls. These results are in accordance with other similar studies [2, $20,21]$. Decreased magnesium serum levels in children with T1DM were reported in the study by Lin et al. [11] but, without the similar decrease of serum zinc levels when compared with healthy controls. Similar serum zinc levels between T1DM and healthy group were also reported in other studies $[22,23]$.

Zn plays a major role in the stabilisation of insulin hexamers and the pancreatic storage of the hormone, and is an efficient antioxidant. Zn may also have an indirect insulin-like effect, with genetic studies identifying the islet-restricted Zn transporter ZnT8 as a likely player in the control of insulin secretion. When the serum $\mathrm{Zn}$ concentration falls, there is a concomitant reduction in insulin secretion and peripheral insulin sensitivity [24]. Ahmed and Helal [20] in their study comprised of 25 children with T1DM and 13 apparently healthy controls, reported decreased magnesium and zinc serum levels in T1DM children compared to the healthy controls. These results could be explained by the fact that magnesium and zinc levels may decrease due to urinary loss by osmotic action, glucosuria and hyperglycemia [20].

With respect to the duration of the disease within the T1DM group, there were no significant differences 
found in this study in serum magnesium, zinc and iron levels. This finding was similar to the study by Estakhri et al. in which zinc serum levels did not correlate to the duration of disease [23]. However, Ahmed and Helal [20] in their study report a negative correlation of serum magnesium and zinc levels with the duration of T1DM. Possible reasons for these contradictory results can be due to the difference in duration of disease between patient populations, quality of glycemic control, genetic, dietary characteristics, and environmental factors [23].

Significantly, in this study, lower zinc serum levels were observed in children with poorer glycemic control. Similarly, decreased levels of serum zinc in children with T1DM and poorer glycemic control were also described in the studies of Alghobashy et al. from 2018 [1] and Lin et al. from 2014 [11]. The negative correlation of serum zinc level with the degree of disease control was also observed by Ahmed and Helal [20], however, the study by Estakhri et al. [23] showed that the level of serum zinc was not influenced by the quality of the glycemic control. Although, this discrepancy may be explained by the fact that the study of Estakhri et al. [23] included a significantly smaller number of children with T1DM $(\mathrm{N}=30)$ whose disease duration was significantly shorter (2.5 years). Lower levels of zinc increase the production of hydroxyl radicals, facilitating oxidative stress in children with T1DM, through the decrease of iron binding to the cell membrane as well as through the inhibition of metallothionens [11]. The negative correlation of zinc levels with $\mathrm{HbA}_{1 \mathrm{c}}$ levels in children with poorer glycaemic control is one observable negative effect of this oxidative injury [2]. Decreased zinc serum levels are also associated with impaired utilisation of glucose and decreased insulin sensitivity [25].

The aforementioned negative correlation of serum $\mathrm{Zn}$ levels with respect to $\mathrm{HbA}_{1 \mathrm{c}}$ level can be explained by increased urinary micronutrient loss due to increased osmotic diuresis caused by hyperglycemia [1]. The absence of a correlation between disease duration and serum $\mathrm{HbA}_{1 \mathrm{c}}$ level is most likely the result of good glycemic control and relatively short average disease duration in our study group of children with T1DM (4.1 years). In contrast, the study results of Al Ghobashy et al. [1] indicate a positive correlation of disease duration with $\mathrm{HbA}_{1 \mathrm{c}}$ levels in children with T1DM. However, the children with T1DM in this study had a mean age of illness of 4.8 years, with $75 \%$ of them defined as having poorly controlled disease.

Zinc supplementations can, through the increase of serum zinc levels, help to decrease diabetes-induced oxidative stress [2]. The fact that zinc levels in this study directly correlated to poorer glycemic control, represented by higher $\mathrm{HbA}_{1 \mathrm{c}^{\prime}}$, seems to further suggest that zinc correction could be beneficial to children with T1DM.

Indeed, several studies, such as Jayarwardena et al. [25] and the meta-analysis by Capdor et al. [26] indicated that the use of zinc supplementation showed a tendency to decrease $\mathrm{HbA}_{1 \mathrm{c}}$ levels in patients with T1DM. Magnesium supplements were administered to children with T1DM during 3 months in the study conducted by Shahbah et al. [27] and were shown to significantly decrease $\mathrm{HbA}_{1 \mathrm{c}}$ levels. Introducing more magnesium and zinc rich food in the diet of children with T1DM probably represents an easy and beneficial method to balance the element's serum levels and improve glycemic control.

While iron supplementation can certainly alleviate the iron deficiency anemia associated with diabetes [14] as well as its possible negative effects on non-verbal intelligence as observed by Mojs et al. [28], there is currently not enough clinical evidence on their use in children with T1DM.

Limitations of this study include its cross-sectional design, as well as the fact that data were collected from a single centre and therefore may not be extrapolate to other population groups.

In conclusion, magnesium and zinc supplementation or nutritional intake could potentially be included in the standard treatment of children with T1DM to achieve better glycemic control, however, further multi-centre, longituidal studies are required. Furthermore, the usage of iron supplementation in diabetic children is currently controversial and also requires further research.

\section{Conflict of interest}

No potential conflict of interest relevant to this article was reported.

\section{REFERENCES}

1. Alghobashy AA, Alkholy UM, Talat MA, et al. Trace elements and oxidative stress in children with type 1 diabetes mellitus. Diabetes Metab Syndr Obes. 2018; 11: 85-92, doi: 10.2147/DMSO. S157348, indexed in Pubmed: 29618936.

2. Özenç S, Saldir M, Sarı E, et al. Selenium, zinc, and copper levels and their relation with $\mathrm{HbA} 1 \mathrm{c}$ status in children with type 1 diabetes mellitus. Int J Diabet Dev Countries. 2015; 35(4): 514-518, doi: 10.1007/s13410-015-0327-y.

3. Zhang H, Forman HJ, Choi J. Gamma-glutamyl transpeptidase in glutathione biosynthesis. Methods Enzymol. 2005; 401 468-483, doi: 10.1016/S0076-6879(05)01028-1, indexed in Pubmed: 16399403.

4. Al Alawi AM, Majoni SW, Falhammar H. Magnesium and human health: perspectives and research directions. Int J Endocrinol. 2018: 1-17, doi: 10.1155/2018/9041694, indexed in Pubmed: 29849626.

5. Praveeena S, Pasula S, Sameera K. Trace elements in diabetes mellitus. J Clin Diagn Res. 2013; 7(9): 1863-1865, doi: 10.7860/ JCDR/2013/5464.3335, indexed in Pubmed: 24179883. 
6. Atabek ME, Kurtoglu S, Pirgon $O$, et al. Serum magnesium concentrations in type 1 diabetic patients: relation to early atherosclerosis. Diabetes Res Clin Pract. 2006; 72(1): 42-47, doi: 10.1016/j.diabres.2005.09.002, indexed in Pubmed: 16214256.

7. Agarwal R, lezhitsa L, Agarwal P. Pathogenetic role of magnesium deficiency in ophthalmic diseases. BioMetals. 2013; 27: 5-18, doi: 10.1007/s10534-013-9684-5, indexed in Pubmed: 24233809.

8. Sakaguchi Y, Shoji T, Hayashi T, et al. Hypomagnesemia in type 2 diabetic nephropathy: a novel predictor of end-stage renal disease. Diabetes Care. 2012; 35(7): 1591-1597, doi: 10.2337/ dc12-0226, indexed in Pubmed: 22498805.

9. Lefebvre B, Vandewalle B, Balavoine AS, et al. Regulation and functional effects of ZNT8 in human pancreatic islets. J Endocrinol. 2012; 214(2): 225-232, doi: 10.1530/JOE-12-0071, indexed in Pubmed: 22582094

10. Marreiro Dd, Cruz KJ, Morais JB, et al. Zinc and oxidative stress: current mechanisms. Antioxidants (Basel). 2017; 6(2): 24, doi: 10.3390/antiox6020024, indexed in Pubmed: 28353636.

11. Lin $\mathrm{CC}$, Huang $\mathrm{HH}, \mathrm{Hu} \mathrm{CW}$, et al. Trace elements, oxidative stress and glycemic control in young people with type 1 diabetes mellitus. J Trace Elem Med Biol. 2014; 28(1): 18-22, doi: 10.1016/j. jtemb.2013.11.001, indexed in Pubmed: 24315963.

12. Urrechaga $\mathrm{E}$. Influence of iron deficiency on $\mathrm{Hb} A 1 \mathrm{c}$ levels in type 2 diabetic patients. Diabetes Metab Syndr. 2018; 12(6): 1051-1055, doi: 10.1016/j.dsx.2018.06.024, indexed in Pubmed: 30042079.

13. Akkermans MD, Mieke Houdijk ECA, Bakker B, et al. Iron status and its association with $\mathrm{HbA} 1 \mathrm{c}$ levels in Dutch children with diabetes mellitus type 1. Eur J Pediatr. 2018; 177(4): 603-610, doi: 10.1007/s00431-018-3104-3, indexed in Pubmed: 29396628.

14. Wójciak RW, Mojs E, Stanisławska-Kubiak M. The occurrence of iron-deficiency anemia in children with type 1 diabetes. J Investig Med. 2014; 62(6): 865-867, doi: 10.1097/ JIM.0000000000000098, indexed in Pubmed: 25011021.

15. Ito $H$, Takeuchi $Y$, Ishida $H$, et al. Mild anemia is frequent and associated with micro- and macroangiopathies in patients with type 2 diabetes mellitus. J Diabetes Investig. 2010; 1(6): 273-278, doi: 10.1111/j.2040-1124.2010.00060.x, indexed in Pubmed: 24843443.

16. Liu Q, Sun L, Tan Yi, et al. Role of iron deficiency and overload in the pathogenesis of diabetes and diabetic complications. Curr Med Chem. 2009; 16(1): 113-129, doi: 10.2174/092986709787002862, indexed in Pubmed: 19149565.

17. Marjanac I, Lovrić R, Barbić J. Serum levels of the high-mobility group box 1 protein (HMGB1) in children with type 1 diabetes mellitus: case-control study. Cent Eur J Immunol. 2019; 44(1): 33-37, doi: 10.5114/ceji.2019.84012, indexed in Pubmed: 31114434.
18. DiMeglio LA, Acerini CL, Codner E, et al. ISPAD Clinical Practice Consensus Guidelines 2018: Glycemic control targets and glucose monitoring for children, adolescents, and young adults with diabetes. Pediatr Diabetes. 2018; 19 Suppl 27: 105-114, doi: 10.1111/pedi.12737, indexed in Pubmed: 30058221.

19. Clinical and Laboratory Standards Institute (CLSI) Procedure for the Collection of Diagnostic Blood Specimens by Venipuncture. Approved Standard. Fifth Edition. Wayne, PA, USA: 2007. CLSI document H3-A5.

20. Ahmed MM, Helal SR. Study of serum magnesium, zinc, copper, and glyco-hemoglobin in children with type 1 diabetes mellitus. Alexandria J Ped. 2002; 16: 285-289.

21. Salmonowicz B, Krzystek-Korpacka M, Noczyńska A. Trace elements, magnesium, and the efficacy of antioxidant systems in children with type 1 diabetes mellitus and in their siblings. Adv Clin Exp Med. 2014; 23(2): 259-268, doi: 10.17219/acem/37074, indexed in Pubmed: 24913117.

22. Zargar AH, Bashir MI, Masoodi SR, et al. Copper, zinc and magnesium levels in type-1 diabetes mellitus. Saudi Med J. 2002; 23(5): 539-542, indexed in Pubmed: 12070576.

23. Estakhri M, Djazayery A, Eshraghian Mr, et al. Serum zinc levels in children and adolescents with type-1 diabetes mellitus. Iran J Public Health. 2011; 40(4): 83-88, indexed in Pubmed: 23113106.

24. Ortega RM, Rodríguez-Rodríguez E, Aparicio A, et al. Poor zinc status is associated with increased risk of insulin resistance in Spanish children. Br J Nutr. 2012; 107(3): 398-404, doi: 10.1017/ S0007114511003114, indexed in Pubmed: 22277170.

25. Jayawardena R, Ranasinghe $P$, Galappatthy $P$, et al. Effects of zinc supplementation on diabetes mellitus: a systematic review and meta-analysis. Diabetol Metab Syndr. 2012; 4(1): 13, doi: 10.1186/1758-5996-4-13, indexed in Pubmed: 22515411.

26. Capdor J, Foster M, Petocz $\mathrm{P}$, et al. Zinc and glycemic control: a meta-analysis of randomised placebo controlled supplementation trials in humans. J Trace Elem Med Biol. 2013; 27(2): 137-142, doi: 10.1016/j.jtemb.2012.08.001, indexed in Pubmed: 23137858.

27. Shahbah D, Hassan T, Morsy S, et al. Oral magnesium supplementation improves glycemic control and lipid profile in children with type 1 diabetes and hypomagnesaemia. Medicine (Baltimore). 2017; 96(11): e6352, doi: 10.1097/MD.0000000000006352, indexed in Pubmed: 28296769.

28. Mojs E, Stanisławska-Kubiak M, Wójciak RW, et al. Reduced iron parameters and cognitive processes in children and adolescents with DM1 compared to those with standard parameters. J Investig Med. 2016; 64(3): 782-785, doi: 10.1136/jim-2015-000054, indexed in Pubmed: 26912011. 